\title{
Reaching the ultimate performance limit given by non-local effects in BOTDA sensors
}

\author{
Alejandro Dominguez-Lopez*a ${ }^{* a}$ Zhisheng Yang ${ }^{\text {bc }}$, Marcelo A. Soto ${ }^{\mathrm{b}}$, Xabier Angulo-Vinuesa ${ }^{\mathrm{a}}$, \\ Sonia Martin-Lopez ${ }^{\mathrm{a}}$, Luc Thevenaz ${ }^{\mathrm{b}}$ and Miguel Gonzalez-Herraez ${ }^{\mathrm{a}}$ \\ ${ }^{a}$ Dept. of Electronics, University of Alcala, Polytechnic School, 28805 Alcala de Henares, Spain; \\ ${ }^{\mathrm{b}}$ Inst. of Electrical Eng., Swiss Federal Inst. of Tech. (EPFL), CH-1015 Lausanne, Switzerland; \\ ${ }^{c}$ State Key Lab. of Inf. Phot. \& Opt. Com., Univ. of Posts and Telecom., 100876 Beijing, China.
}

\begin{abstract}
Non-local effects have been traditionally identified as one of the most limiting factors of the performance of Brillouin optical time-domain analyzers. These phenomena, directly linked with the energy gained/lost by the pump pulse, limit the probe power and ultimately the SNR of the system. Several solutions have been proposed, although none offers the possibility to increase the probe power until its limit, the onset of amplified spontaneous Brillouin scattering. In this work, we propose a technique that avoids non-local effects and permits to set the probe power at its maximum, reaching a $100 \mathrm{~km}$ sensing distance with 2 meter resolution.
\end{abstract}

Keywords: Brillouin distributed sensors, Brillouin scattering, nonlinear optics, fiber optic sensors.

\section{INTRODUCTION}

Brillouin Optical Time Domain Analysis (BOTDA), a consolidated long-range distributed fiber optic sensing technique, traditionally uses a Dual-Probe-Sideband (DSB) modulation scheme ${ }^{1}$, which turns the system to be highly robust against pump depletion and non-local effects compared to a Single Sideband (SSB) probe wave configuration ${ }^{2}$. Even though this procedure works effectively in most of the cases, a recent study ${ }^{3}$ has demonstrated that DSB configuration introduces a spectral distortion on the pump pulse that leads to Brillouin Frequency Shift (BFS) determination errors. This phenomenon scales with the probe wave power, which limits the performance of the BOTDA system in terms of signalto-noise ratio (SNR) and distance. In this work, we propose a novel pump-probe frequency scanning technique that completely eliminates non-local effects ${ }^{3}$, up to the ultimate probe power limit given by the onset of amplified spontaneous Brillouin scattering (ASpBS).

\section{PRINCIPLE}

In principle, by making use of a DSB configuration, the maximum probe power can be significantly raised in the sensing fiber to be ultimately limited to $\sim 6-7 \mathrm{dBm}$ by the onset of $\mathrm{ASpBS}^{2}$. This scheme works perfectly when the gain spectrum is exactly located at the median position between the two sidebands, however, it has been recently found ${ }^{3}$ that such scheme still suffers from non-local effects when probe and gain spectrum are detuned, even for power in each sideband far below the ASpBS threshold. By sweeping the frequency of the two sidebands in the traditional way ${ }^{1}$, as shown in Fig. 1(a), the pump pulse spectrum is subject to an uneven spectral compensation of the gain-loss probe spectra in case of detuning. The pulse spectrum turns out then to be asymmetrically distorted and downshifted or upshifted as the pulse propagates along the sensing fiber, depending on whether the scanned pump-probe frequency offset is higher or lower than the average BFS of the fiber ${ }^{3}$. This detrimental effect comes from the non-zero net gain (indicated by green lines in Fig. 1(a)) that combines Brillouin gain (red curves) and loss (blue curves) spectra generated by the upper and lower probe sidebands, respectively. This pulse distortion actually cumulates along the sensing fiber, inducing a severe distortion in the gain/loss BOTDA traces, thus giving rise to errors in the BFS estimation. The longer the sensing fiber is and the higher the probe power used, the more distorted the pump pulse is.

Instead of sweeping the two probe sidebands symmetrically away and towards the pulse spectrum ${ }^{1-3}$, a novel sweeping method is proposed in this paper. This technique keeps a fixed frequency separation between the two probe sidebands while sweeping pump or probe wave frequency to scan the Brillouin Gain or Loss Spectrum (BGS or BLS), as shown in Fig. 1(b). This way, the gain and loss spectra generated by the two probe sidebands exactly cover the same spectral region and mutually cancel out, regardless of the scanned pump-probe frequency offset. Consequently, the pulse

*alejandro.dominguezl@uah.es

24th International Conference on Optical Fibre Sensors, edited by Hypolito José Kalinowski, José Luís Fabris, Wojtek J. Bock, Proc. of SPIE Vol. 9634, 96342E · C 2015 SPIE

CCC code: $0277-786 \mathrm{X} / 15 / \$ 18 \cdot$ doi: $10.1117 / 12.2205440$

Proc. of SPIE Vol. 9634 96342E-1 
spectrum experiences no spectral distortion during propagation and Brillouin interaction. As demonstrated hereafter, this proposed technique allows the probe power to reach the ultimate limit given by the ASpBS threshold, representing about $10 \mathrm{~dB}$ enhancement on probe power (and consequently on SNR) when compared to the traditional probe configuration.

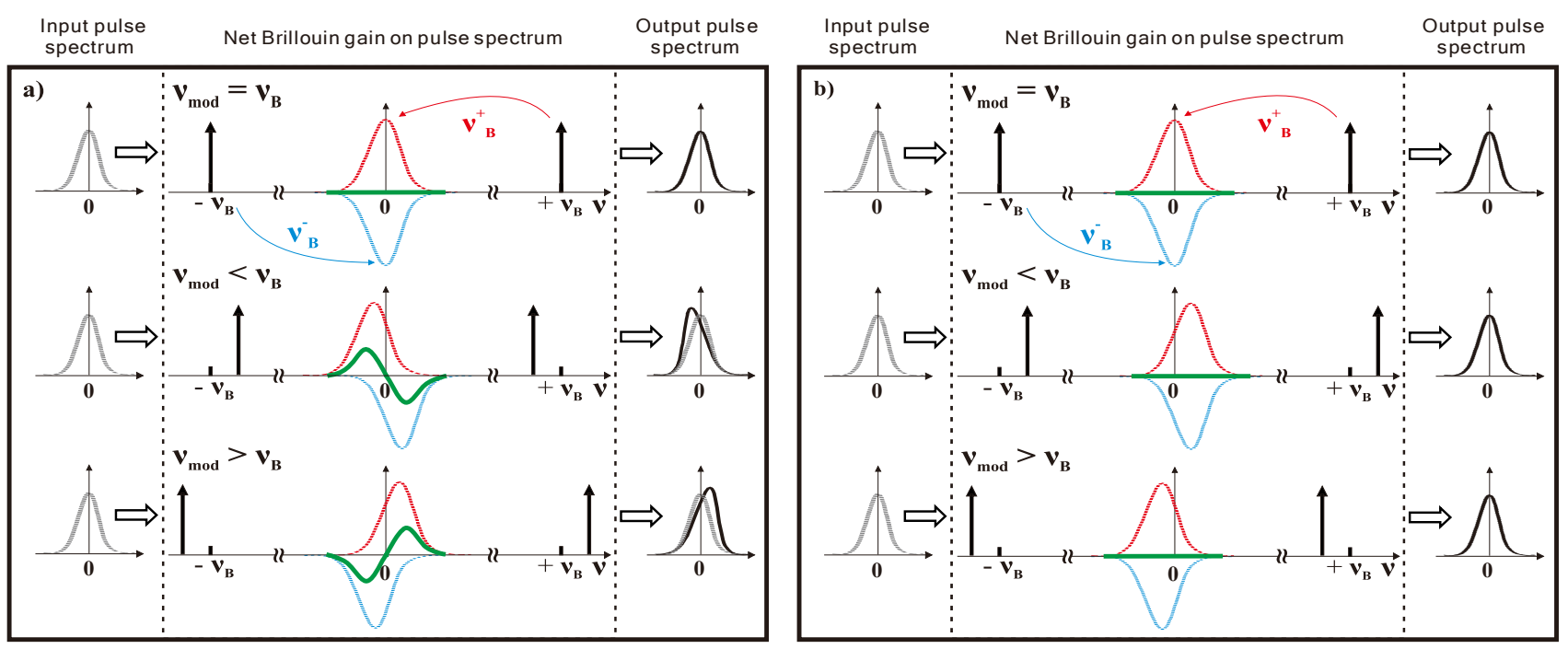

Fig. 1. Net Brillouin gain depleting the pump pulse while scanning the pump-probe frequency offset. (a) Scanning symmetrically around the pulse spectrum (traditional scheme). (b) Proposed scanning keeping a fixed frequency separation between sidebands. Grey dashed lines: input pulse spectrum; red dashed lines: gain spectrum generated by the upperfrequency probe sideband; blue dashed lines: loss spectrum generated by the lower-frequency probe sideband; green solid lines: net Brillouin gain spectrum experienced by the pulse; black solid line: output pulse spectrum after SBS interaction.

\section{RESULTS}

The proposed scanning method, presented in Fig. 2, is a modification of a standard BOTDA scheme ${ }^{3}$ although, in this case, the probe wave remains fixed in the spectrum and the pump pulse is the one that sweeps to properly scan the Brillouin gain or loss processes. First of all, the laser light is DSB-modulated using an intensity Electro-Optic Modulator (EOM1 in Fig. 2), whose frequency is set to match the average BFS $\left(v_{\mathrm{B}}\right)$ of the fiber. Afterwards, the modulated Continuous Wave (CW) is split into two branches; one already constitutes the probe wave and is directly launched into the Fiber Under Test (FUT). The other one is used to generate the pump pulse. For this purpose, it is first necessary to select either the Stokes (Brillouin gain) or anti-Stokes (Brillouin loss) modulation sidebands by means of a suitable filter. In this case a Dense Wavelength Division Multiplexer (DWDM) is used to select the Stokes band, which is amplitude modulated by means of a second modulator (EOM2 in Fig. 2) at a frequency difference sweeping around the BFS ( $v_{\mathrm{B}} \pm$ $\Delta v)$. This new frequency component, which provides the pump wave, is selected through a narrow tunable filter ( $\sim 148 \mathrm{pm}$ of bandwidth), wide enough to allow a frequency tuning along the selected spectral span and narrow enough to filter out all unwanted spectral components. Subsequently, the signal is pulsed through a Semiconductor Optical Amplifier (SOA), and then amplified by an Erbium Doped Fiber Amplifier (EDFA) and de-polarized by means of a Polarization Scrambler (PS) in order to avoid polarization fading in the measured temporal traces.

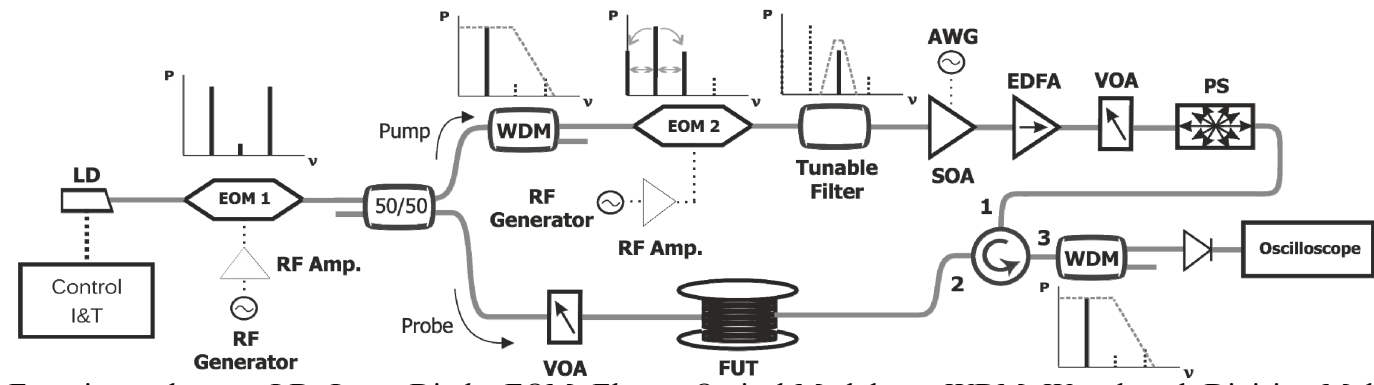

Fig. 2: Experimental setup. LD: Laser Diode; EOM: Electro-Optical Modulator; WDM: Wavelength Division Multiplexer; AWG: Arbitrary Waveform Generator; SOA: Semiconductor Optical Amplifier; EDFA: Erbium Doped Fiber Amplifier; RF: Radio-frequency generator; VOA: Variable Optical Attenuator; PS: Polarization Scrambler; FUT: Fiber Under Test. 
Now we will take a look at the results obtained using the proposed solution. It should be noted that all the measurements have been performed for a spatial resolution of $2 \mathrm{~m}$ ( $20 \mathrm{~ns}$ pulse width). As previously stated, the pump pulse suffers a huge spectral distortion when the frequency of the probe wave is swept as in standard BOTDA systems. Such spectral deformation grows dramatically when the power of the probe wave is considerably increased ${ }^{3}$. Fig. 3i.a shows the BGS and BLS retrieved at the very end of a $25 \mathrm{~km}$ Single Mode Fiber (SMF) spool when the probe wave is swept through the conventional method and for a probe power level of $\sim 0 \mathrm{dBm} /$ sideband and a pulse peak power of $\sim 12.4 \mathrm{dBm}$. As a previous work has reported ${ }^{3}$, a distorted BLS is obtained in this case, while the BGS shows minimum distortion. If the probe power is below $-6 \mathrm{dBm}$, a safe scenario is guaranteed, where no distortion is experienced. Beyond this limit, distortion starts to arise, particularly in the BLS case. When increasing the probe power up to $\sim 4.8 \mathrm{dBm}$, the distortion obtained in both BGS and BLS is massively increased, as shown in Fig. 3ii.a, being essentially affected by the appearance of two high-power side lobes. Considering that no distortion arises at the central frequency $(\sim 10.74 \mathrm{GHz}$ in this case), it is remarkable the unexpected huge amplitude of these two new spurious peaks, which for the BLS case (Fig. 3ii.a) can reach up to one order of magnitude higher than the amplitude at the central frequency. Such a distortion in the BGS and BLS conceals any eventual temperature/strain variation along the fiber, making this probe power impractical.

Contrarily to the use of the standard configuration, when the proposed sweeping method is applied under the same pump and probe power conditions shown before, the Brillouin gain and loss profiles measured at the end of the fiber show no distortion at all. In Fig. 3i.b and Fig. 3ii.b, it can be observed that both the BGS and BLS follow the usual Lorentzian shape and present expected gain/loss values (which remain consistent when increasing the probe power). It should be emphasized that, in this case, any eventual temperature/strain non-homogeneity occurring at the end of the fiber would be determined correctly, as it will be demonstrated in forthcoming paragraphs. This new sweeping method ensures correct measuring conditions and permits increasing the probe power in at least $\sim 10 \mathrm{~dB}$ (from $-6 \mathrm{dBm}$ to $\sim 4.8 \mathrm{dBm}$ ), over the standard BOTDA scheme, being only limited by the ASpBS threshold of the fiber. Such an increase raises considerably the system SNR $(+10 \mathrm{~dB})$ and enhances the overall system performance, including the sensing range.
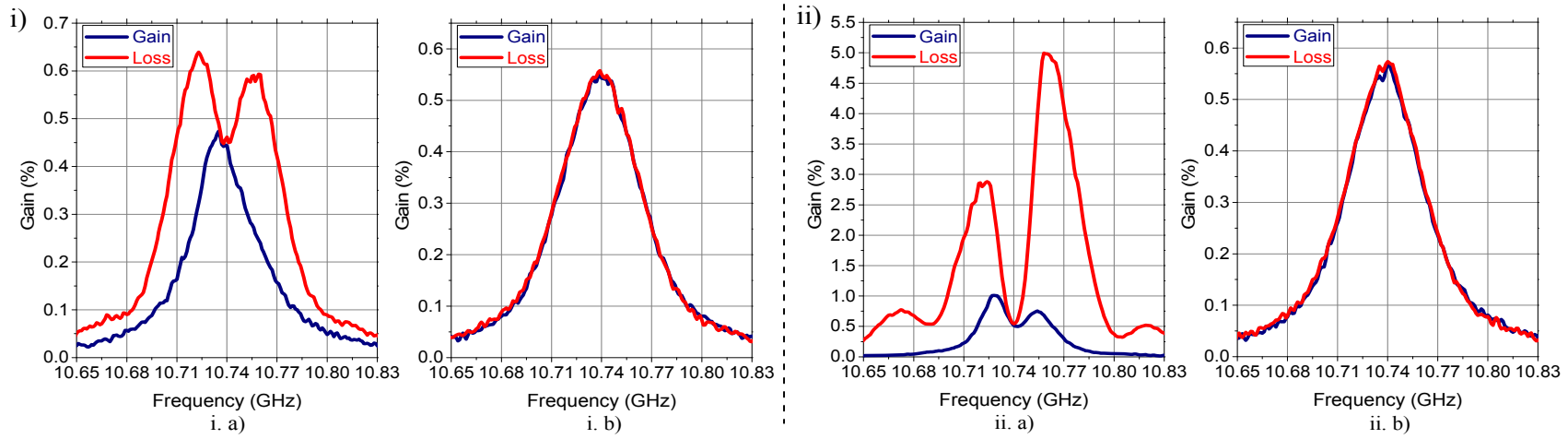

Fig. 3: Illustration of the Brillouin Gain and Loss spectra at $\mathrm{km} 24.970$ for a $\sim 25 \mathrm{~km}$ SMF spool for different probe powers (Case i: $\sim 0 \mathrm{dBm}$ probe power; case ii: $\sim 4.8 \mathrm{dBm}$ probe power). a) Conventional DSB-BOTDA scanning method. b) Novel scanning method proposed, where the pump scans the Brillouin gain and loss for fixed probe wave sidebands.

Making use of the improved sensing performance demonstrated before for the proposed scheme and owning to the increased probe power, measurements along $100 \mathrm{~km}$ with $2 \mathrm{~m}$ resolution have been possible. Fig. 4a shows the maximum BOTDA amplitude trace of the $100 \mathrm{~km}$ fiber span for a pump-probe frequency shift of $\sim 10.856 \mathrm{GHz}$. As can be observed, the fiber end is perfectly visible, showing a good contrast with the background noise (SNR of $\sim 6 \mathrm{~dB}$ at the fiber end). The pump pulse peak power employed in this case is $\sim 13.7 \mathrm{dBm}$ and the probe wave is $\sim 4.8 \mathrm{dBm}$ (similar to the case in Fig. 3ii.a), while each time trace has been averaged 16000 times.

To further verify the performance of the implemented sensor, a 5 meter hot-spot has been placed at the end of the fiber (around $100 \mathrm{~km}$ ) in a hot water bath at $\sim 60^{\circ} \mathrm{C}$ (being $\sim 25^{\circ} \mathrm{C}$ room temperature). A full frequency sweep is performed to retrieve the BFS change at the hot-spot position; the result of the temperature change around the hot-spot is shown in Fig. $4 \mathrm{~b}$. A $\sim 35{ }^{\circ} \mathrm{C}$ change over room temperature is verified in the hot-spot location (considering a linear relation temperature-frequency $\approx 1{ }^{\circ} \mathrm{C} / \mathrm{MHz}$ ), which matches the real temperature difference. The sensing uncertainty (estimated as the standard deviation of the repeatability in 5 consecutive measurements) around the hot-spot is $2.8^{\circ} \mathrm{C}$. In addition, the spatial resolution of the system has been verified to be $2 \mathrm{~m}$, which has been determined by analyzing the slope length of the detected hot-spot. Moreover, the hot-spot is correctly identified as being $\sim 5 \mathrm{~m}$ long. In terms of Figure of Merit ${ }^{6}$ (FoM), the improvement obtained by using this configuration is a factor 10, which follows the $10 \mathrm{~dB}$ SNR improvement 
accomplished. It should be noted that this result has been achieved without using any extra technique, such as Raman assistance $^{4}$ or pump pulse coding ${ }^{5}$, which implies a brand new measuring performance record. The simplicity of the proposed setup makes these results even more impressive when compared with other studies reaching similar distances.

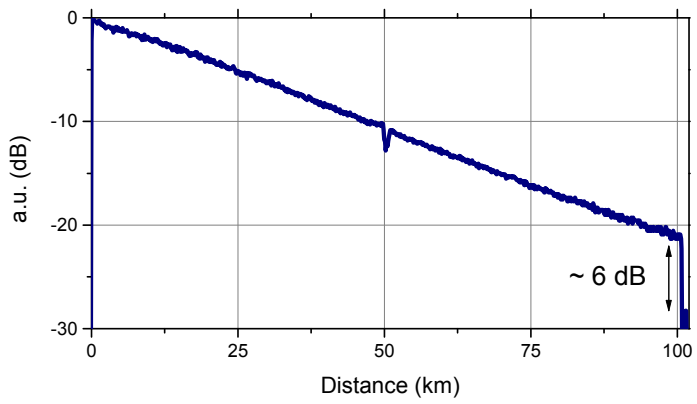

a)

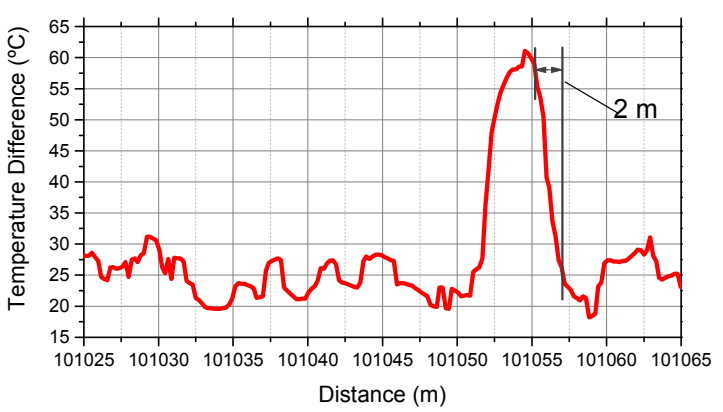

b)

Fig. 4: a) BOTDA trace represented in logarithmic scale of the $100 \mathrm{~km}$ FUT for a pump-probe frequency shift of 10.856 $\mathrm{GHz}$, averaged 16000 times. b) BFS translated to temperature difference for a $\sim 5$-meter hot-spot located around $\mathrm{km} 100$.

\section{CONCLUSIONS}

In conclusion, we have presented a novel Brillouin optical time-domain analysis technique highly robust against pump depletion and non-local effects. This allows increasing the probe wave power level until the appearance of ASpBS, which is translated into a sensor performance improvement. The measuring methodology is based on varying the pumpprobe modulation structure by just fixing the probe sidebands detuning to match the average Brillouin frequency shift of the fiber, and then sweeping the pump pulse frequency. In this case, the recovered Brillouin gain and loss curves are not distorted by non-local effects. Of course, matching the proper pump-probe frequency shift of the fiber is critical to ensure correct measurements. In these conditions, it is possible to increase the probe power up to the ASpBS threshold, allowing us to measure, without any extra assistance, over $100 \mathrm{~km}$ with 2 meter resolution (16000 averages) and $2.8 \mathrm{MHz}$ uncertainty. The proposed scheme allows a FoM improvement factor of 10 over the traditional sweeping scheme. This FoM improvement is directly related to the $10 \mathrm{~dB}$ SNR improvement accomplished. We believe this technique brings a considerable upgrade for BOTDA systems, giving the possibility of combining it with other additional techniques, such as Raman assistance or pulse coding. We foresee that, combining all these techniques, it might be potentially feasible to measure up to $150 \mathrm{~km}$ of fiber with similar resolution values (2 meters).

\section{ACKNOWLEDGEMENTS}

This work was supported in part by the European Research Council through StG U-FINE (Grant 307441), in part by the Spanish MINECO under Projects TEC2012-37958-C02-01, TEC2012-37958-C02-02, and TEC2013-45265-R; the INTERREG SUDOE program ECOAL-MGT and the Comunidad de Madrid under projects EDISON (CCG2014/EXP072), and SINFOTON-CM:S2013/MIT-2790. Z. Yang acknowledges the China Scholarship Council for supporting his stay at the EPFL. The work of SML was supported by the Spanish MINECO through a "Ramón y Cajal" Contract.

\section{REFERENCES}

[1] Nikles, M., Thévenaz, L., and Robert, P. A., "Brillouin gain spectrum characterization in single-mode optical fibers," J. Lightwave Technol., 15(10), 1842-1851 (1997).

[2] Thévenaz, L., Mafang, S. F., and Lin, J. "Effect of pulse depletion in a Brillouin optical time-domain analysis system" Opt. Express 21(12), 14017-14035 (2013).

[3] Domínguez-López, A., Angulo-Vinuesa, X., López-Gil, A., Martín-López, S., and González-Herráez, M., "Non-local effects in dual-probe-sideband Brillouin optical time domain analysis" Opt. Express (23) 8, 10341-10352 (2015).

[4] Angulo-Vinuesa, X., Martin-Lopez, S., Nuño, J., Corredera, P., Ania-Castañon, J. D., Thévenaz, L., and González-Herráez, M., "Raman-assisted Brillouin distributed temperature sensor over $100 \mathrm{~km}$ featuring 2 meter resolution and $1.2^{\circ} \mathrm{C}$ uncertainty," $\mathrm{J}$. Lightwave Technol. 30(8), 1060-1065 (2012).

[5] Soto, M. A., Bolognini, G., and Di Pasquale, F., "Long-range simplex-coded BOTDA sensor over $120 \mathrm{~km}$ distance employing optical preamplification," Opt. Lett. 36(2), 232-234 (2011).

[6] Soto, M. A., and Thévenaz, L., "Modeling and evaluating the performance of Brillouin distributed optical fiber sensors" Opt. Express, 21(25), 31347-31366 (2013). 